\title{
Tratamiento del Flujo Genital *
}

Comentario a ciento treinta casos de clientela privada

\author{
Dr. Eduardo Acosta Bendek
}

Médico-Jefe del Servicio de Maternidad "Leopoldina de Santos"

Barranquilla, Noviembre 20 de 1959

Ha sido consagrada por el uso la palabra Leucorrea, que etimológicamente quiere decir flujo blanco, como sinónimo de flujo genital. Flujo genital significa toda pérdida por vagina, no hemática, cualquiera fuere su color; razón por la cual prefiero el nombre de flujo genital y no de Leucorrea.

El flujo genital puede tener su origen en cualquier sector del tracto genital, pero sólo me referiré en este trabajo, al flujo cervico-vaginal.

El material para este estudio fué tomado de mi consulta privada, y comprende ciento treinta casos de edades y tipo de pacientes diferentes, que consultaron por flujo genital, prurito, e irritación de genitales externos. A todas las pacientes se les instruyó no usar ducha vaginal, ni relaciones sexuales por 72 horas antes del examen. Las muestras se tomaron del cérvix, y fondo de saco vaginal posterior, y en muy pocos casos de meato y orina, con aplicadores estériles, los cuales se colocaban en sus respectivos tubos de ensayos también esterilizados, que contenían un centímetro de solución salina fisiológica. Las muestras eran llevadas inmediatamente al laboratorio para ser examinadas por los métodos siguientes:

Examen microscópico directo en fresco entre lámina y laminilla para verificar presencia de leucocitos, Trichomonas y ele-

* Contribución al tema oficial de Flujos presentado al IV Congreso Colombiano de Obstetricia y Ginecología 1959. 
mentos de hongos. Preparación coloreada (Gram) para investigar bacterias, principalmente bacilo de Doederlein y hongos.

Siembras en medios de cultivo. - Se usaron cinco medios de cultivo diferentes a saber: Agar-sangre, para gérmenes cocoides, Haemofilus, Corynebacterium. Proteosa $N^{0} 3$ agar con hemoglobina, para estos mismos gérmenes y especialmente para Gonococo.

Manitol-sal-agar, para Estafilococo y sus características bioquímicas. McConkey-agar, para gérmenes del grupo Coliforme, Salmonellas y Shigellas. Sabouraud dextrosado (con Cloramfenicol) para Candida albicans y la mayoría de los demás hongos.

Incubación de los medios de cultivo sembrados a $37^{\circ} \mathrm{C}$. durante 24 y 48 horas, para el informe preliminar, y durante seis días para el informe definitivo. En algunos casos se incubó en atmósfera con $10 \%$ de $\mathrm{CO}_{2}$.

El estudio cualitativo de la flora vaginal encontrado, se puede observar en el cuadro siguiente:

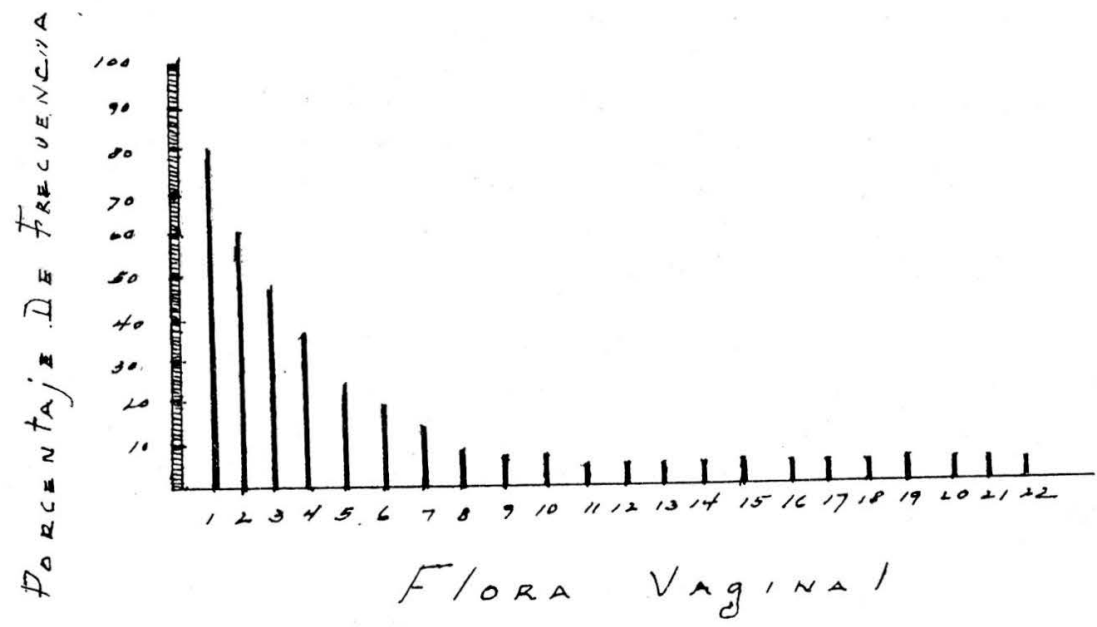

$N^{o}$ de casos

1-Bacilo Doederlein ... . . . ... ............ 101

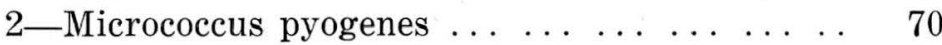

$\begin{array}{lllllllll}3-\text { Monilia albicans } & \ldots & \ldots & \ldots & \ldots & \ldots & \ldots & \ldots & 58\end{array}$

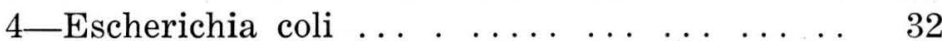

$\begin{array}{lllllll}5 \text {-Trichomonas vaginalis } & \ldots & \ldots & \ldots & \ldots & \ldots & 27\end{array}$ 
6-Corynebacterium difteroide .. . . . . . . . . 16

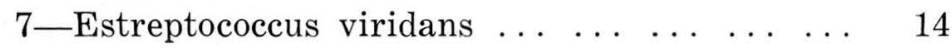

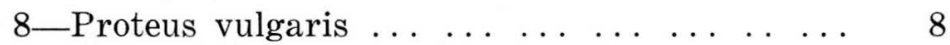

$\begin{array}{lllllll}9 \text {-Estreptococcus faecalis } & \ldots & \ldots & \ldots & \ldots & \ldots & 6\end{array}$

10-Estreptococcus beta-hemolitic . . . . . . . . . 5

11 -Bacilus Proteus . . . . . . . . $\ldots \ldots \ldots \ldots \ldots$

12-Estreptococcus v. gama $\ldots \ldots \ldots \ldots \ldots \ldots$

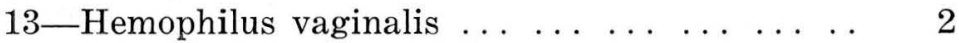

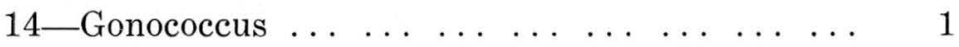

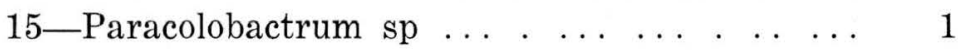

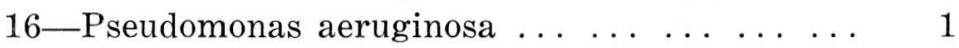

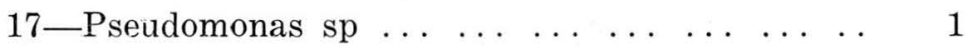

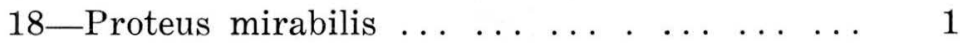

19-Hongo género Monilia no albicans ... . . . . 1

20-Hongo género Trichophyton $\ldots \ldots \ldots \ldots \ldots$

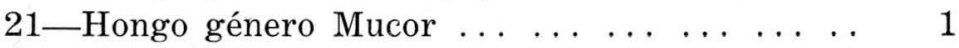

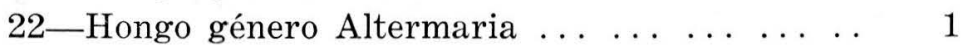

De los 130 casos de flujo genital sólo 5 de ellos fueron de origen psicógeno y hormonal, el resto fué de tipo infeccioso. El grado de pureza vaginal fué de tipo II y III en el $77.69 \%$ y tipo grado IV en $\in 118.46 \%$. De las vaginitis específicas, la monilia albicans ocupó el primer lugar, y se aislaron tres géneros diferentes de hongos registrándose un caso de cada uno de ellos, y otro del género Monilia, pero no albicans.

La Trichomona vaginalis siguió en orden de importancia, con la salvedad de que no se hicieron cultivos para Trichomonas por no haber sido fácil conseguir uno de los componentes de dicho medio; sólo se hizo el diagnóstico por examen microscópico directo en fresco.

De las vaginitis no específicas, la variedad de microorganismos presentes en la flora vaginal fué numerosa, siguiendo en orden de frecuencia e importancia el Micrococcus pyogenes, el Escherichia coli, el Corynebacterium difteroide y los Estreptococcus. Con relación del Hemophilus vaginalis, su poca frecuencia registrada se debió a que fueron pocos los casos en que se hizo el cultivo selectivo para éste. Sobre la importancia que algunos (1) le han dado al $\mathrm{H}$. vaginalis como causa de las vaginitis no específicas, no es aceptada aún como tal por otros investigadores (2). La 
implicación que puedan tener el resto de microorganismos aislados como causantes de estas vaginitis es aún motivo de discusión; sin embargo el M. pyogenes estuvo presente en casi todos los casos de vaginitis.

Bret y colaboradores (3) señalan la importancia de la frecuencia del M. pyogenes en la Trichomoniasis vaginal, hecho que hemos comprobado. En estudios realizados en pacientes normales se ha encontrado la presencia de Trichomonas y Monilia albicans, sin que hayan causado molestia alguna de flujo genital o vaginitis, lo que nos hace pensar que existen otros factores coadyuvantes en la producción de éstas.

Sólo un caso de Gonococo fué reportado en una paciente gestante; la frecuencia de éste como causa de vaginitis ha decrecido notoriamente desde la era de los antibióticos, al paso que las vaginitis a Monilia cada día son más frecuentes.

\section{ASOCIACION DE GERMENES:}

Microorganismos

asociado a la Monilia

$N^{o}$ de Casos

$\%$

Monilia Albicans

y Trichomonas Vaginalis

Monilia Albicans

y Micrococcus pyogenes

Monilia Albicans

y Escherichia Coli

Monilia Albicans

y Estreptococcus Viridans

10

7.69

\section{Microorganismos}

asociados a la T. vaginalis

$N^{o}$ de Casos

$\%$

T. vaginalis y Monilia albicans

$\mathrm{T}$. vaginalis y Micrococcus pyogenes

T. vaginalis y Escherichia Coli

T. vaginalis y Estreptococcus viridans

T. vaginalis y Estreptococcus faecaelis 
Se observa en los cuadros anteriores la presencia de Trichomonas y Monilia conjuntamente en cinco casos, el germen más frecuentemente asociado tanto en la Moniliasis como en la Trichomoniasis, fué el M. pyogenes, siguiendo en orden de frecuencia el Escherichia coli y el estreptococcus viridans. Algunos investigadores (4) atribuyen mayor importancia a la asociación de la T. vaginalis y el Estreptococcus fecaelis, que a la de M. pyogenes, en tanto que en nuestras observaciones encontramos sólo esta asociación en un caso.

Actualmente las infecciones producidas por la $\mathrm{T}$. vaginalis $\mathrm{y}$ la M. albicans, no se hallan completamente esclarecidas, ya que aún están por dilucidar los factores que intervienen en ellas. Es un hecho real que la presencia de la T. vaginalis y de la M. albicans no son sinónimos de vaginitis. Entre las causas coadyuvantes se han invocado las asociaciones de bacterias o de hongos, $\mathrm{pH}$ €'ievado o bajo, posible presencia de toxinas etc. En nuestras observaciones hemos encontrado como dato muy diciente que la mayor incidencia de estas vaginitis (bien sean específicas o no) se registran en mujeres con antecedentes obstétricos y en la edad de mayor actividad sexual, que para nuestro modo de pensar son de las principales causas coadyuvantes.

INCIDENCIA POR EDAD:

\begin{tabular}{rcr}
\hline Edad & $N^{o}$ de pacientes & $\%$ \\
\hline $1-10$ & 4 & 3.07 \\
$10-20$ & 12 & 9.23 \\
$20-30$ & 72 & 55.38 \\
$30-40$ & 27 & 20.75 \\
$40-50$ & 8 & 6.15 \\
$50-60$ & 2 & 1.53 \\
\hline
\end{tabular}

En el cuadro anterior llama la atención que el mayor número de las pacientes infectadas, correspondió al grupo de edad entre 20 y 30 años, el segundo lugar al grupo entre los 30 y 40 años; edades que representan la época de mayor actividad sexual en la mujer, lo cual nos hace valorar la importancia del coito como factor coadyuvante o desencadenante en la producción del flujo genital. El grupo correspondiente a las niñas y al de la menopausia, es muy reducido. 
TIPO DE PACIENTES:

\begin{tabular}{lcc}
\hline Pacientes & $N^{o}$ de Casos & $\%$ \\
\hline Obstétricas & 36 & 27.69 \\
Ginecológicas & 94 & 72.31 \\
\hline
\end{tabular}

De las 36 pacientes obstétricas registradas, la vaginitis más frecuente fué la de Monilia albicans con 22 casos, la Trichomoniasis vaginal se encontró en 6 casos; el gonococo en 1 caso, los casos restantes correspondieron a las vaginitis no específicas. La vaginitis a Monilia albicans fué tan frecuente como la vaginitis no específica en las pacientes ginecológicas, siguiéndole en su orden las vaginitis o trichomonas vaginalis. Entre estas pacientes hubo 2 menopáusicas, y 8 vírgenes, entre las cuales había 4 niñas; el resto correspondió a mujeres casadas.

Tratamiento: Al abordar el tema sobre el tratamiento y con el fin de ser un poco didáctico para su estudio, he querido hacerlo de acuerdo con la edad y tipo de paciente. Es bueno puntuaiizar como principio del tratamiento de toda vaginitis, los hechos siguientes :

Eliminación del parásito, restauración normal de la vejiga, y por último prevenir las recaídas y reinfecciones.

El tratamiento que he usado en los pocos casos de vaginitis en las niñas, consistió en la eliminación del microorganismo aislado con Furacín o Quemicetina en su forma de candelillas; de acuerdo a la sensibilidad registrada en el antibiograma, asociado a la administración oral del Dietilestilbestrol así:

$0.1 \mathrm{mg}$. durante 15 días y control citológico vaginal. Los resultados fueron satisfactorios en general.

En la mujer casada para mejor éxito del tratamiento, se aconsejó la prohibición de relaciones sexuales mientras se llevaba a término éste. Como rutina general acostumbro a practicar limpieza completa de vagina y cérvix; si la vaginitis es a Trichomonas hago insuflación de polvos Furazolidona (Tricofurona) o del ácido acetilamino-hidroxifenil-arsónico (picatyl) diario durante tres días, seguido de una tableta vaginal mañana y noche de estos mismos productos durante catorce días; se hace control tres días 
después y se ordena continuar con la aplicación por cuatro ciclos más, de una tableta durante los siete días siguientes al período seguido de nuevo control. Si presentare recaída o reinfección se repetirá el tratamiento, complementado con el control del esposo y tratamiento a éste llegado el caso. Cuando las Tricomonas se encuentran en útero, uretra y vejiga, o en la próstata en los hombres, el tratamiento se dificulta notablemente por cuanto las drogas administradas por vía oral como tricomonacidas no han dado los resultados esperados. Mientras no se produzca una droga de mayor efectividad por ingesta, el tratamiento de las Tricomonas vaginales será incompleto.

RESULTADO DEL TRATAMIENTO

DE LAS VAGINITIS A TRICOMONAS

\begin{tabular}{lcccc}
\hline & $\begin{array}{c}\text { No de } \\
\text { pacientes }\end{array}$ & $\begin{array}{c}\text { No de } \\
\text { curadas }\end{array}$ & Recurrencia & Fracasos \\
\hline $\begin{array}{l}\text { T. Vaginalis } \\
\text { T. Vaginalis y }\end{array}$ & 22 & 15 & 5 & 2 \\
\begin{tabular}{l} 
M. albicans \\
\multicolumn{1}{c}{ Total }
\end{tabular} & 5 & 2 & 1 & 2 \\
\hline
\end{tabular}

En la vaginitis a Monilia, se utilizó la solución acuosa de violeta de genciana al 5\% en embrocaciones día por medio, oscilando entre 3 y 8 , seguidas de la aplicación local de una tableta de Micostatin mañana y noche por siete días; se hace control tres días más tarde y luego se continúa con una tableta nocturna por catorce días más y se vuelve hacer nuevo control. En los casos de recaídas o reinfecciones se repite el tratamiento asociándolo al micostatin oral.

RESULTADO DEL TRATAMIENTO

DE LAS VAGINITIS A M. ALBICANS

\begin{tabular}{|c|c|c|c|c|}
\hline & $\begin{array}{c}\mathrm{N}^{0} \text { de } \\
\text { pacientes }\end{array}$ & $\begin{array}{l}\mathrm{N}^{0} \mathrm{de} \\
\text { curadas }\end{array}$ & Recurrencia & Fracasos \\
\hline $\begin{array}{l}\text { Monilia albicans } \\
\text { Monilia albicans y } \\
\text { Tricomona } \\
\text { vaginalis }\end{array}$ & 53 & 38 & 5 & 10 \\
\hline Total & 58 & 42 & 6 & 10 \\
\hline
\end{tabular}


En las vaginitis no específicas se usaron preferentemente, óvulos de Quemicetina, de nitrofurazona (furacín) y gantrisin crema vaginal diario durante 15 días, seguidos de control 3 días después. Se utilizaron con más frecuencia las sulfas y nitrofurazona por razón de haber observado que los antibióticos, desarrollaban en algunos casos vaginitis a Monilias. Los resultados obtenidos fueron satisfactorios.

\section{RESULTADO DEL TRATAMIENTO \\ DE LAS VAGINITIS NO ESPECIFICAS}

\begin{tabular}{|c|c|c|c|c|}
\hline & $\begin{array}{c}\mathrm{N}^{0} \mathrm{de} \\
\text { pacientes }\end{array}$ & $\begin{array}{l}\mathrm{N}^{\mathbf{0}} \text { de } \\
\text { curadas }\end{array}$ & Recurrencia & Fracasos \\
\hline $\begin{array}{l}\text { E. coli, M. pyo- } \\
\text { genes, Estrepto- } \\
\text { coccus, etc. }\end{array}$ & 44 & 29 & 8 & 7 \\
\hline
\end{tabular}

El único caso de gonorrea fué tratado con terramicina oral y local con curación completa.

En las pacientes menopáusicas además del tratamiento específico se asociaron los estrógenos obteniendo así mejores resultados.

En los casos de flujo genital asociado a cervicitis crónica, se trató ésta por medio de cauterizaciones, en número de una o dos con intervalos de tres semanas, además se complementó con la aplicación intramuscular de la asociación de penicilina-estreptomicina.

\section{COMENTARIOS}

El buen éxito de todo tratamiento de flujo genital está determinado por su diagnóstico inicial, y para que éste dé garantía, es necesario que se haga simultáneamente examen microscópico directo y cultivos selectivos de la muestra tomada; incluyendo aún las Trichomonas vaginalis, ya que hoy en día se ha comprobado que el cultivo es el método más sensible para el diagnóstico de ésta, evitando así muchos resultados negativos falsos.

El tratamiento de las Trichomonas vaginalis es hasta el momento deficiente o incompleto, mientras no se produzca una droga de mayor efectividad por vía oral que las que actualmente existen. 
A propósito de las Tricomonas vaginales somos del parecer, como han sugerido otros especialistas, que habiéndose demostrado la presencia de ésta tanto en el tracto genital superior, como urinario, debe cambiársele el nombre de Trichomonas vaginalis por el de tricomonasis sexual humana o simplemente Trichomonas Donne, nombre de su descubridor.

Las vaginitis a M. albicans se registran como la molestia más frecuente del flujo genital. La causa de la alta incidencia es de todos conocida y consiste preferentemente en el uso indiscriminado de los antibióticos, en especial de los de amplio espectro.

El $90 \%$ de los casos registrados de vaginitis a Monilia albicans acusaban entre sus antecedentes el uso de antibióticos. Los resultados obtenidos en el tratamiento de estas vaginitis a base de violeta de Genciana y micostatin fueron completamente satisfactorios.

Queda aún por demostrar cuáles son los factores que determinan que tanto la Tricomonas vaginales y la M. albicans se vuelvan patógenas.

En lo que respecta a la epidemiología, es aceptado que la $\mathrm{T}$. vaginalis puede ser transmitida durante el coito, sin ser éste el único método de transmisión, ya que se obtiene también por medios extra-sexuales en niñas y jóvenes, como son las contaminaciones por el uso de sanitarios, especialmente entre las personas con una higiene pobre, como también la contaminación del recién nacido por el paso a través del canal del parto.

La epidemiología de la M. albicans es completamente diferente al de las Tricomonas vaginales, ya que se le aisla de la boca, piel y heces, lo que hace que la infección vaginal aparezca por la inoculación del material procedente de estas fuentes.

Lo que hace relación a las vaginitis no específicas está aún en discusión la importancia del Hemophylus vaginalis como causa esencial de ella.

En el tratamiento de estas vaginitis debe dársele preferencia al uso de las sulfas y nitrofurazonas con el fin de evitar las moniliasis consecuentes.

El flujo genital en las pacientes embarazadas, no deja de tener sus riesgos tanto para la madre como para el feto. Ultimamente se han registrado casos de infección amniótica (5) por Monilia albicans, septicemia y estados de shok por E. coli (6). 
Davis y Grand señalan también la importancia de la inflamación crónica de la $\mathrm{T}$. vaginales como contribución a la producción del carcinoma cérvical. (7)

El tratamiento del flujo genital, cualquiera que sea su etiología, ofrece frecuentes dificultades ya que entran en juego una serie de factores independientes del tratamiento en sí, como son el incumplimiento por parte de la paciente y la poca cooperación de los esposos.

\section{CONCLUSIONES :}

1 - De los 130 casos de flujo genital registrados, sólo 5 no fueron de tipo infeccioso.

2 - Se registró la mayor frecuencia de flujo genital, en las pacientes con antecedentes obstétricos, y en la edad de mayor actividad sexual.

3 - El método más sensible de diagnóstico de flujo genital es el cultivo.

4 - Entre las vaginitis específicas la M. albicans registró ser la más frecuente y la E. coli en las no específicas.

5 - La patogenicidad de la $T$. vaginales y la $M$. albicans no está aún esclarecida.

6 - El tratamiento de las T. vaginalis es aún incompleto mientras no se produzca un mejor tricomonacida por vía oral.

7 - La importancia del $\mathrm{H}$. vaginalis como causa de las vaginitis no específica está aún en discusión.

\section{BIBLIOGRAFIA}

1 BRIWER JOHN L., HALPERN BERNARD, THOMAS GARY: Hemophilus Vaginalis Vaginitis. Americans J. Obst. and Gynec. 74. 834, 843, 1957.

2 HELTON ANDRE, TALEGHANY PARVIZ: Nonspeciie Vaginal infectons. American J. Obst. and Gynec. 77, 144, 148, 1959.

3 GRSENHULL J. P. Year book of Obstetrics and Gynecology. 419, 422, 1958. 1959. Chicago.

4 GONZALEZ RAMOS M.: Peróxido de Hidrógeno en el tratamiento de las Trichomoniasis Vaginal. Ginec. Obst. de México. Nov, Dic. 1955. No 6. 
5 BEDOYA J. M., FERNANDEZ ORTEGA J. M.: Resultados del tratamiento oral de la tricomoniasis sexual humana con dos amino-5mitrotiazol (tritheon) y tricomicina. Rev. Española Obst. y Ginec. XVII 97, 1, 3, 1958.

6 BEMISCHKE KURT, RAPHAEL SUMMER L.: Cándida albicans infection of the anmiotic Sac. American J. Obst. and Gynec. 75, 200, 202, 1958.

7 OXORN HARRY. Shock not related to blood loss, with Escherichia coli, Septicemia in the Obstetric patient. American J. Obst. and Gynec. 78, 567, 569, 1959.

8 DAVIS, C. H., and GRAND, C. C. 1952. Vaginalis Donne and evaluation experimental and clinical data. American J. Obst. and Gynec. 64, 544, 548, 1952.

9 BIERCH THOMAS A., REES CHARLES W., REARDON LUCY V.: Epidemiological studies on human trichomoniasis. American J. Trop. med. e Higien. 8: 312 318. 1959.

10 KARNAKY KARL JOHN: Comparison of Battery $\mathrm{pH}$ readings and Electronic $\mathrm{pH}$. Recordings of the vagina in Trichomonas vaginalis infestation. American J. Obst. and Gynec. 77, 149, 154, 1959.

11 ENSEY JAMES E.: Treatment of Vulvovaginitis with a new combination of Nitrofurans. Americans J. Obst. and Gynec. 77, 155, 158. 1959.

12 WOLINSKA WANDA H.: The value of a prepared culture medium in the diagnosis Trichonomas vaginalis. American J. Obst. and Gynec. 77, 306, 308, 1959.

13 BURCH THOMAS A., REES CHARLES W., REARDON LUCY, BETHESDA. Diagnosis of Trichomonas vaginalis Vaginitis. American J. Obst. and Gynec. 77, 309, 313, 1959.

14 AMAYA LEON HERNANDO: Tricomoniasis vaginal. Comentarios a una modificación terapéutica. Rev. Col. Obst. y Gynec. IX, 539, 545, 1958.

15 BEDOYA J. M. RICO L. R.: Factores hipofuncionales ováricos en la tricomoniasis sexual en la mujer. Frecuencia de la infección en menopáusicas. Rev. Española Obst. Ginec. XVI, 1, 5, 1957.

16 SEREBRINSKY JULIO: Flujo Genital. Prensa Médica Argentina. XIL 233, 239, 1958.

17 RUBIN and NOVAK: Integrated Gynecology. I 563, 568, 1956. Mc Graw Hill-New York.

18 SALABER JUAN B. A. Ginecología Geriátrica. López \& Etchegoyen, S. R. L. Buenos Aires.

19 CALATRONI CARLOS J. RUIZ VICENTE: Terapéutica Ginecológica. El Ateneo. Florida, 340 Buenos Aires 1946.

NOTA: Expreso mis agradecimientos al Doctor Florentino Rey Matiz, por la colaboración prestada en el estudio bacteriológico del presente trabajo. 